\title{
Sono-magnetic heating in tumor phantom
}

\author{
Katarzyna Kaczmarek ${ }^{1,{ }^{*}}$, Tomasz Hornowski ${ }^{1}$, Iryna Antal ${ }^{2}$, Michal Rajnak ${ }^{2}$, Milan Timko $^{2}$, Arkadiusz \\ Józefczak ${ }^{1, * *}$ \\ ${ }^{1}$ Institute of Acoustics, Faculty of Physics, Adam Mickiewicz University, Uniwersytetu Poznańskiego 2, 61-614 \\ Poznań, Poland \\ 2 Institute of Experimental Physics, Slovak Academy of Sciences, Watsonova 47, Košice 040 01, Slovakia \\ *e-mail: katarzyna.kaczmarek@amu.edu.pl \\ **e-mail: aras@amu.edu.pl
}

\begin{abstract}
There exist various types of hyperthermia therapy such as radiofrequency, infrared, microwave, focused ultrasound or magnetic. Recently, a lot of effort has been put into combining more than one mode of heating into one treatment. The multimodal hyperthermia proves a better alternative in comparison with a single one. In this paper, we show that the application of dual sono-magnetic heating (ultrasound and magnetic together) gives better results than using either of them alone. The advantage of this bimodal treatment lies not only in cumulative heating of target volume (tumor) but also in synergistic interaction between the two mechanisms - the ultrasound sonication can improve the thermal effect of magnetic hyperthermia through the unblocking Brown's relaxation. Furthermore, the ultrasound and magnetic heating are complementary to each other. The temperature rise caused by ultrasound is fast changing and by magnetic field slow changing. So the parameters of ultrasound can serve as coarse-tuning settings of heating while the parameters of the magnetic field as fine-tuning enabling more precise hyperthermia.
\end{abstract}

Keywords: sono-magnetic heating, magnetic nanoparticles, ultrasound, phantom.

\section{Introduction}

Nanoparticles, including magnetic ones, find more and more applications in medicine. Superparamagnetic nanoparticles can be used as nanocarriers for different therapeutic agents (nanomedicines) [1]. Most often they are administered in the form of an aqueous suspension - magnetic fluid, to avoid undesirable effects that may occur after administration of bare magnetic nanoparticles. Magnetic nanomaterial in the form of a bare powder allows for possibility of aggregation and creation of larger structures. Such agglomerates are the cause of undesirable ultrasound backscattering during ultrasound sonication, but can also activate responses of immune systems [2,3]. Nanoparticle suspensions are often use in targeted drug release. Nanoparticles act as a vehicles that carry medicines, where drug release can be triggered by temperature increase. Drug carriers are designed to release their payload at the temperature (e.g., $42-43^{\circ} \mathrm{C}$ ) a few degrees above the physiological temperature [4]. A lot of effort has been put into combining chemotherapy with thermal treatment [5-7]. To be more specific, the temperature rise in the body triggers physiological changes responsible for the weakening of the unhealthy cells. It increases vascular permeability and blood flow, which is helpful for chemotherapy and increases blood oxygenation and oxygen content in tissues, thus sensitizing them for radiotherapy [8]. New methods of heat generation are constantly 
being sought and currently existing are constantly being optimized to enhance the delivery of nanomedicines. For this purpose, it is possible to use a new method: sono-magnetic heating. The presence of magnetic particles in tissue (tumor) increases the ultrasonic attenuation. The nanoparticles work as sonosensitizing materials in the case of ultrasound-induced heating [9]. Superparamagnetic iron oxide nanoparticles are also used for induction of heat by an externally applied AC magnetic field magnetic heating [10]. Because nanoparticles have the dual ability to act as both magnetic and sonosensitizer agents, magnetic and ultrasonic heating may work synergistically to produce a more efficient treatment [11]. Such combination of different therapies allows to use lower dosage of potentially harmful (especially in high concentrations) drugs, substances, and nanoparticles. It can also reduce the time of therapy and magnitude of heating power which can result in the minimization of negative side effects $[5,12,13]$. The promising first results of sono-magnetic heating [11] are encouraging for further research in this area.

The main aims of this work are to study the heating process induced by the AC magnetic field (AMF) and focused ultrasounds (FUS) in the presence of iron oxide nanoparticles and the combined effect resulting from magnetic and ultrasound heating acting simultaneously. For this purpose, we use a tissue-mimicking tumor phantom. It consists of stiff agar phantom embedded with nanoparticles (to model the tumor) surrounded by less stiff pure agar phantom (healthy tissue).

\section{Materials and methods}

\subsection{Nanoparticles fabrication}

Dextran magnetic fluid (DEX-MF) was prepared using the slightly modified Molday procedure [14]. Briefly, $38 \mathrm{~g}$ of dextran was dissolved in $150 \mathrm{ml}$ of water while $10 \mathrm{~g}$ of $\mathrm{FeCl}_{3} \cdot 6 \mathrm{H}_{2} \mathrm{O}$ and $4.2 \mathrm{~g}$ of $\mathrm{FeCl}_{2} \cdot 4 \mathrm{H}_{2} \mathrm{O}$ were dissolved in $26 \mathrm{ml}$ of $2 \mathrm{M} \mathrm{HCl}$. Reacting mixture consisting of ferrous chloride and ferric chloride with dextran polymers of 150000 molecular weight was put to the water bath and heated to $60-65^{\circ} \mathrm{C}$ under mixing. $150 \mathrm{ml}$ of $7.5 \%$ ammonium hydroxide was added dropwise and the mixing continued for another $20 \mathrm{~min}$ at $65^{\circ} \mathrm{C}$. Agglomerates were removed by centrifugation at $5000 \mathrm{rpm}$ for $45 \mathrm{~min}$.

The hydrodynamic particle size distribution of magnetic nanoparticles (MNPs) was measured using a dynamic light scattering (DLS) with a scattering angle of $173^{\circ}$. Temperature trend measurements in the particle size were carried out with the same method. For this, a sample was prepared at room temperature and the Z-average was determined during the heating of the sample inside the measuring device with a temperature-controllable cell. The measurement was performed 3 times at a heating rate of $1^{\circ} \mathrm{C}$ per 5 min ranging from 20 to $90^{\circ} \mathrm{C}$. The magnetization curve was measured using a vibrating sample magnetometer (VSM) installed on a cryogen-free superconducting magnet from Cryogenic Ltd. The ultrasonography images of the tumor phantom were obtained with the system USG (Sonoline Prima, SIEMENS).

\subsection{Agar gel-based tumor model}

Tissue-mimicking phantoms are widely used to study various treatment methods e.g., the hyperthermia and thermal ablation therapy [15-17]. Custom made phantoms, with specific shapes and properties, offer the possibility to conduct experiments in conditions similar to the human body. We choose to use agar-based phantoms since they possess the acoustical properties similar to those of real tissues [9, 18-21]. Agar powder was dissolved in hot water or in a mixture of hot water and magnetic fluid and then leave to cool down, see Fig. 1 [22, 23]. Tissue-mimicking phantom with tumor 
model was formed using a cylindrical phantom with magnetic nanoparticles (mimicking a tumor loaded with magnetic nanoparticles) surrounded by a pure agarose gel (mimicking healthy tissue). Phantom with nanoparticles (tumor model) has the cylindrical shape with $1.0 \mathrm{~cm}$ diameter, $2.0 \mathrm{~cm}$ height and volume about $3 \mathrm{ml}$. The concentration of the agar in phantom, $\phi_{A}$, was $5 \%(\mathrm{w} / \mathrm{w})$ and the concentration of the magnetic nanomaterial, $\phi_{M}$, was approximately $0.33 \%(w / w)$. The concentration of pure agarose gel surrounding tumor model was $4 \%(w / w)$. The volume of the whole phantom containing the tumor model was about $25 \mathrm{ml}$. Owing to the fact that healthy tissues and tumors have different mechanical properties [24] - tumors exhibit higher stiffness and lower elastic moduli than normal tissues [25-27] - our tumor model has a higher concentration of agar (higher stiffness) comparing to the surrounding pure agar phantom (lower stiffness). For the preparation of agar-gel phantom with MNPs we used agar powder characterized by the company HiMedia as a standard plate count agar (Standard Methods Agar M091-500G) and magnetic nanoparticles.

A
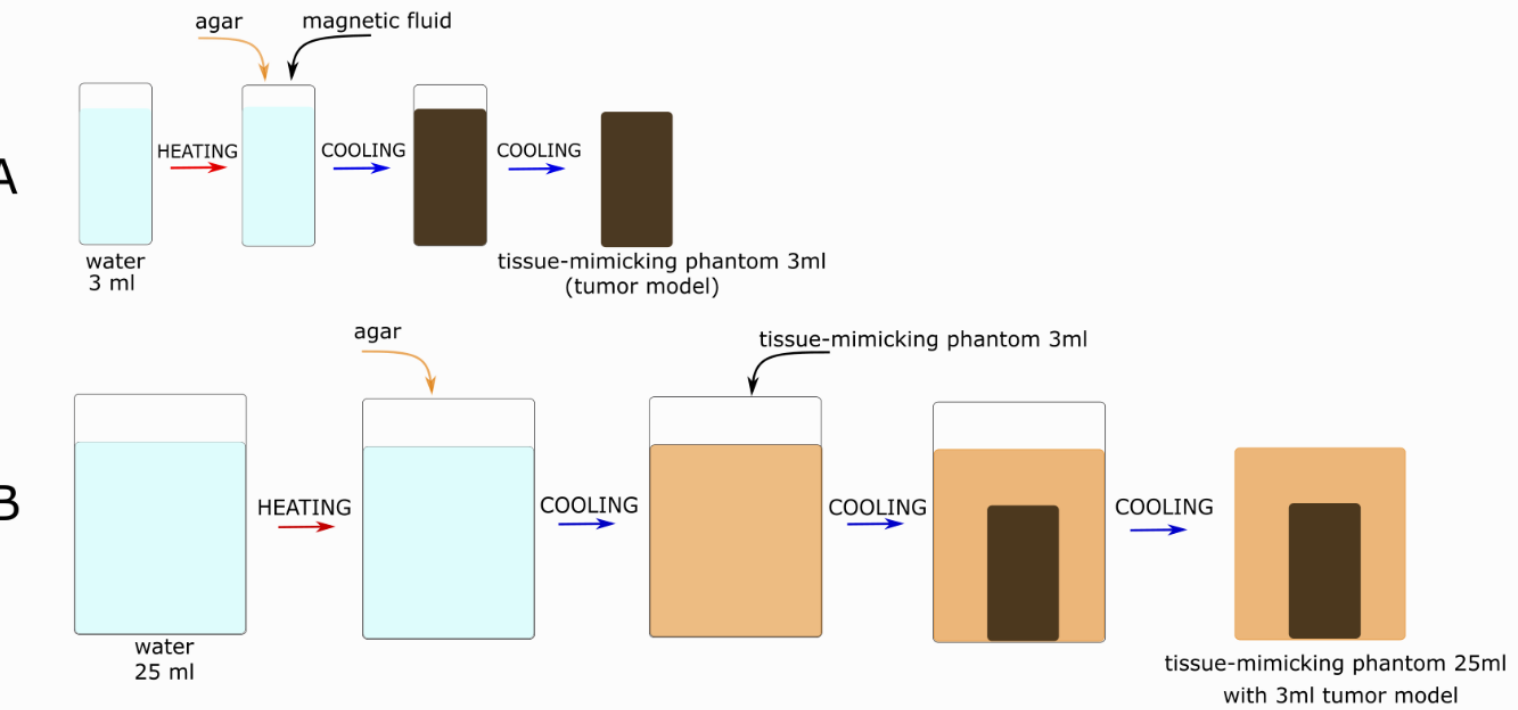

Fig. 1. Preparation of tissue-mimicking phantom: A) $3 \mathrm{ml}$ tissue-mimicking phantom embedded with MNPs (tumor model), B) $25 \mathrm{ml}$ tissue-mimicking phantom with placed inside $3 \mathrm{ml}$ tumor model.

\subsection{Sono-magnetic heating system}

The measuring system for sono-magnetic heating consisted of two parts: the magnetic heating system (EASYHEAT, Ambrell Corporation) with an induction coil and the ultrasound heating system (Optel Ltd.) with a transducer emitting focused ultrasound (Fig. 2). The induction coil had a length of $60 \mathrm{~mm}$ and five turns of 56-mm diameter. The frequency of AMF was equal to $356 \mathrm{kHz}$, and the intensity was in the range of $5.2-16.2 \mathrm{kA} / \mathrm{m}$. The ultrasound transducer worked in continuous mode with the operating frequency of $1 \mathrm{MHz}$ and acoustic power ranging from 0.6-1.4 W. The acoustic power was estimated using the radiation force balance method [28]. In order to position the thermometer as close as possible to the focus of the ultrasound beam, the phantom (containing the optical fiber) was mounted on a tri-axis positioning system $(x, y, z)$. To locate the ultrasound focus point the phantom was manually moved by steps of $1 \mathrm{~mm}$, along the $x, y, z$-axis in order to find localization $(x, y z)$ of the highest temperature rise (ultrasound focus). During experiments the phantom was exposed to either alternating magnetic field or focused ultrasound, and simultaneously to AMF and FUS. The ultrasonic 
transducer, induction coil and the phantom were immersed in degassed and distilled water at room temperature. The temperature variations induced during this sono-magnetic experiments were registered by a digital thermometer (FLUOTEMP, Photon Control Inc.) with optical probe placed inside the tumor phantom.

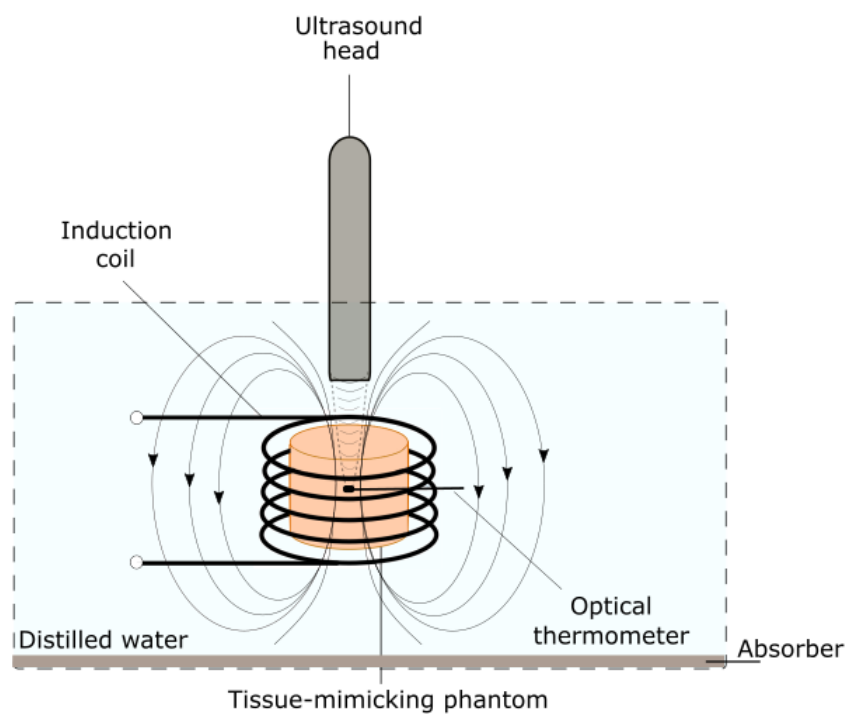

Fig. 2. The measurement setup for sono-magnetic heating.

\section{Results and discussion}

\subsection{Nanoparticles characterization}

The particle size distribution (PSD) in the initial magnetic fluid (non-diluted) as obtained from the DLS measurement is shown in Fig. 3a together with the dependence of the average hydrodynamic diameter, $d_{\text {hyd, }}$ and polydispersity index (PDI) on temperature (Fig. 3b). It can be seen that temperature does not affect the values of $d_{\text {hyd }}$ and PDI so the preparation of phantoms in high temperature does not change the particle size distribution.
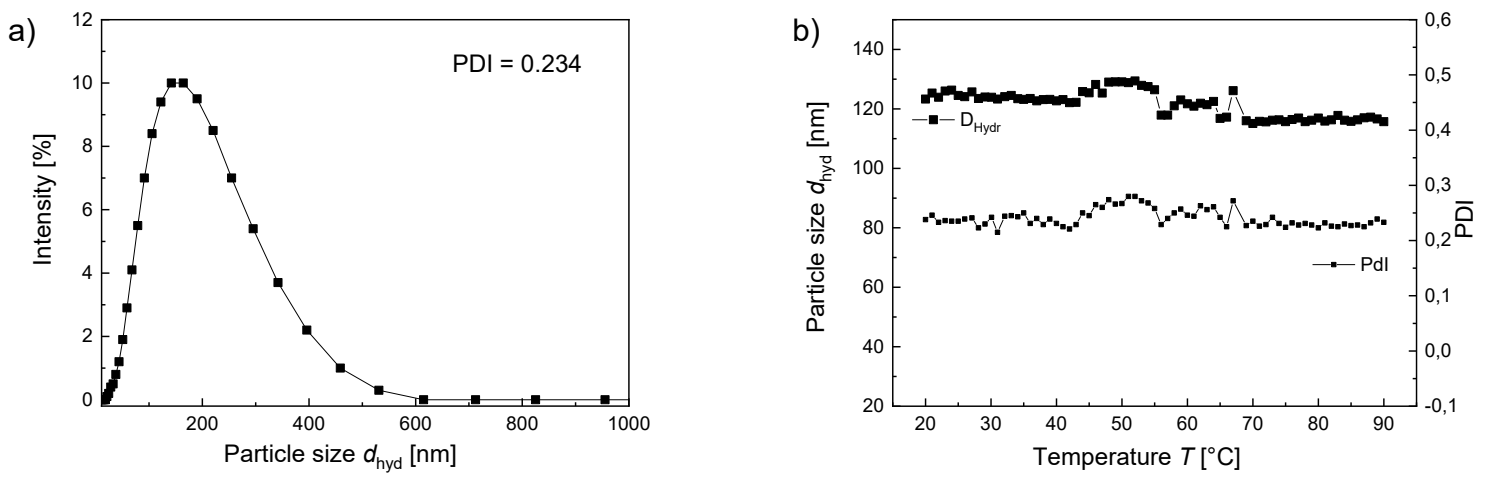

Fig. 3. a) Intensity size distribution of the hydrodynamic diameter $d_{\text {hyd }}$ (Dynamic Light Scattering (DLS) measurements). b) Mean hydrodynamic diameter and polydispersity index PDI in the function of temperature. 
The DC-magnetization curves in water-based magnetic fluids used to prepare the agar phantoms with different concentrations of nanoparticles are shown in Fig. 4a whereas the magnetization curves of the agar phantoms embedded with magnetic nanoparticles are presented in Fig. 4b. The results show that the magnetic saturation of magnetic fluids is slightly smaller than that of agar phantoms embedded with magnetic nanoparticles, despite the same concentrations of magnetic nanoparticles in both cases, e.g., $0.22 \mathrm{emu} / \mathrm{g}$ for magnetic fluids with $0.9 \%$-concentration of magnetic nanoparticles and $0.26 \mathrm{emu} / \mathrm{g}$ for the agar phantom prepared using the same magnetic fluid and doped with the same amount of nanoparticles. This trend is seen for all concentrations of magnetic nanoparticles and can be explained as follows: the magnetic nanoparticles locked in the gel are more resistant to thermal movements that disturb the magnetic moment orientation along the magnetic field direction and increase the value of saturation magnetization.

The well-known method to estimate particles size distribution from VSM measurements requires the finding of the best fit of the volume averaged-Langevin function with the lognormal PSD of the form

$$
f(x)=\frac{1}{\sqrt{2 \pi} x S} \exp \left[-\frac{\ln ^{2}\left(x / D_{0}\right)}{2 S^{2}}\right]
$$

to experimental magnetization data, where $D_{0}$ and $S$ are the parameters of the distribution. Then the mean particle diameter $d_{m}$ and its standard deviation $\sigma$ can be evaluated from the formula $d_{m}=D_{0} \exp (S / 2)$ and $\sigma=D_{0}\left(\exp S^{2}-1\right)^{1 / 2}$ [29]. The results are shown in Fig. 5. It should be noted that the nanoparticle size determined from magnetization measurement is the magnetic core size. It is always less than hydrodynamic size obtained from DLS measurement by the thickness of additional layer/layers surrounding magnetic particle. The average size of the magnetic grain is virtually the same in MF and agar phantom confirming that the process of preparing the phantom does not change the granular properties of magnetic nanoparticles. The solid lines in Fig. 4 present the magnetization curves predicted theoretically with PSDs shown in Fig. 5.

a)

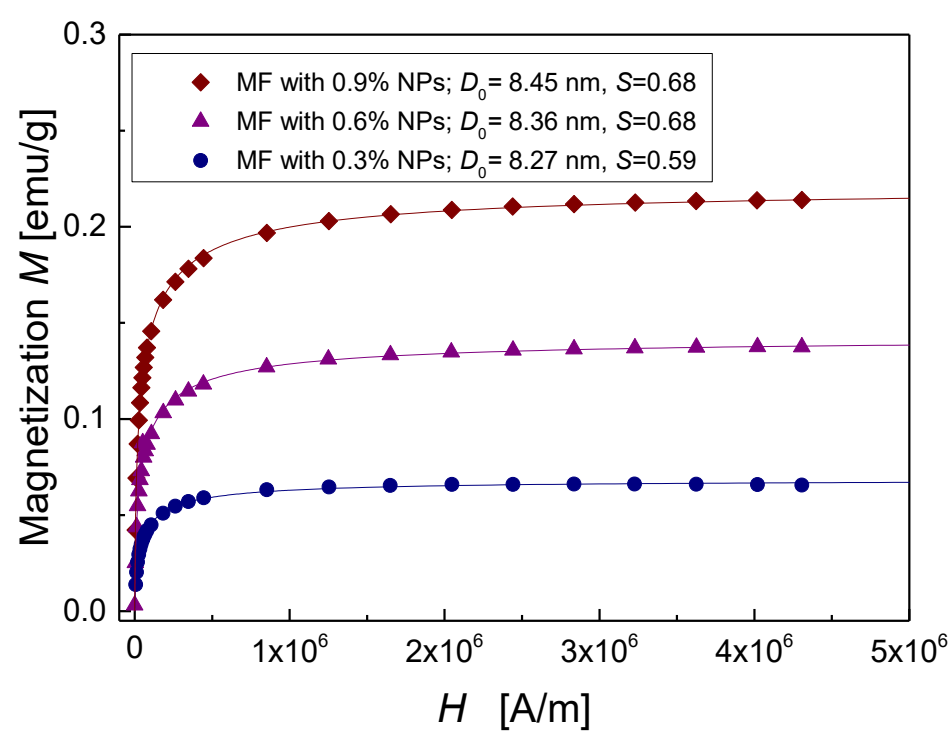

This is a peer-reviewed, accepted author manuscript of the following article: Kaczmarek, K., Hornowski, T., Antal, I., Rajnak, M., Timko, M., \& Józefczak, A. (2020). Sono-magnetic heating in tumor phantom. Journal of Magnetism and Magnetic Materials , 500, [166396]. https://doi.org/10.1016/j.jmmm.2020.166396 
b)

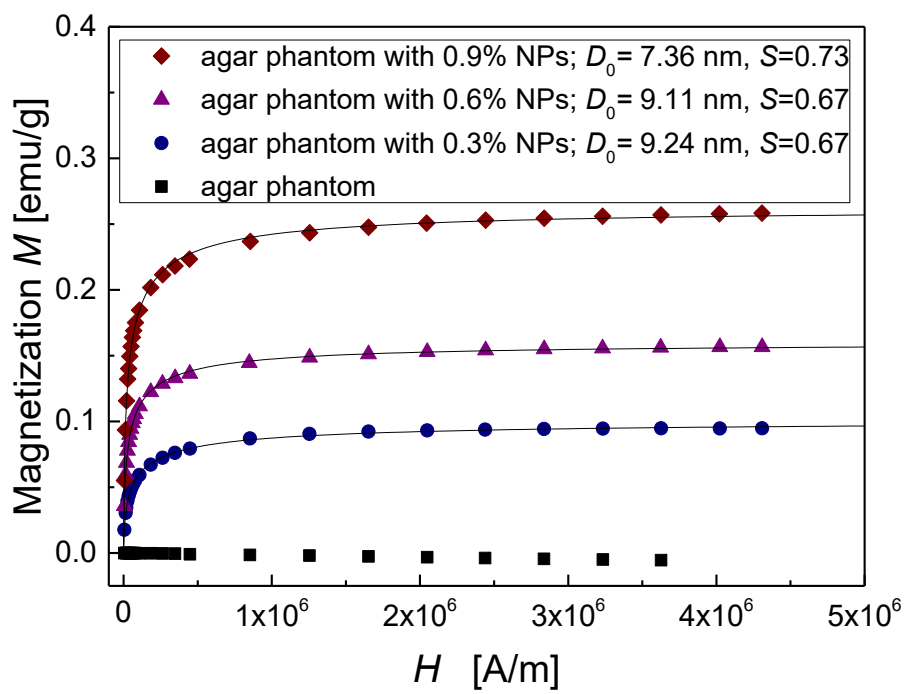

Fig.4. Magnetization curves of a) magnetic fluid (MF) and b) pure agar phantom and agar phantoms with magnetic nanoparticles. The solid lines present the theoretical predictions calculated with the magnetic lognormal size distribution with the parameters shown in Fig. 5.

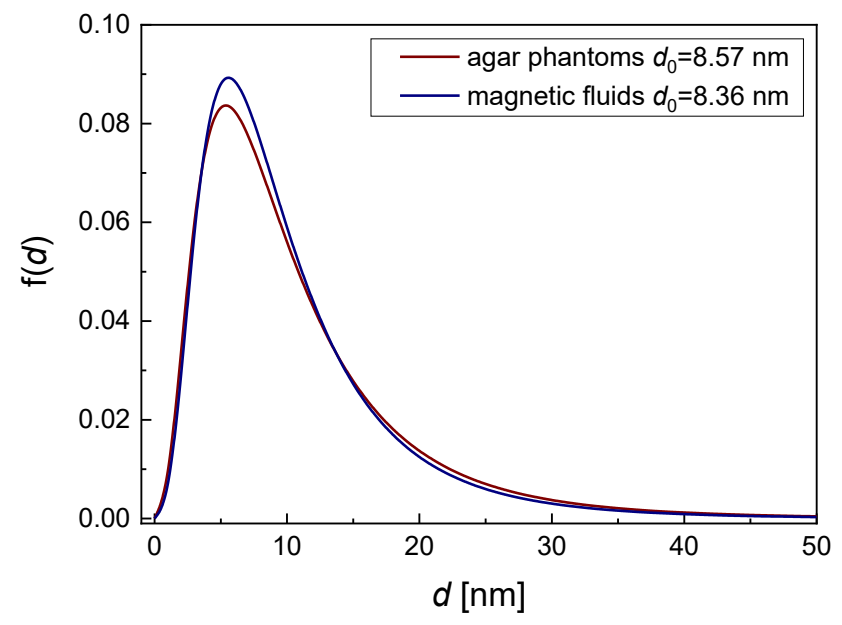

Fig. 5. Magnetic core size distribution obtained from magnetization curves of magnetic fluids and phantoms

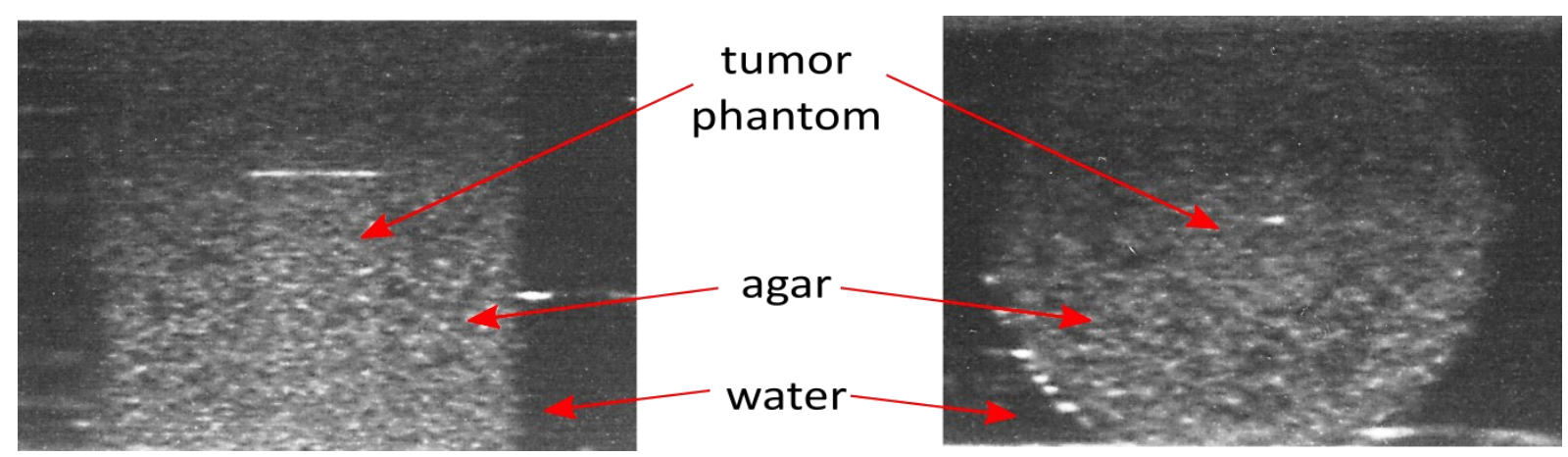

This is a peer-reviewed, accepted author manuscript of the following article: Kaczmarek, K., Hornowski, T., Antal, I., Rajnak, M., Timko, M., \& Józefczak, A. (2020). Sono-magnetic heating in tumor phantom. Journal of Magnetism and Magnetic Materials , 500, [166396]. https://doi.org/10.1016/i.jmmm.2020.166396 
Fig. 6. Ultrasound images of the tumor phantom placed in a cuvette filled with water; side view on the left, top view on the right.

The studied tumor phantom was scanned with ultrasonography (USG). Fig. 6 presents the ultrasound images of the prepared phantom placed in a cuvette filled with water. The images show clearly the boundaries between the water and the agar gel and between the agar and the agar with nanoparticles. The fragment containing the magnetic nanoparticles exhibits higher echogenicity (the largest brightness) because of the higher acoustic impedance $Z=c \cdot \rho(c-$ ultrasound velocity, $\rho-$ density) $[20,30]$. The boundary of the gel with nanoparticles is visible (the intensity of the reflected echo is greater). The prepared phantom simulates the tumor well.

\subsection{Ultrasonic and magnetic heating}

Temperature increase that appears in biological tissues during ultrasound sonication can be explained by the mechanism of ultrasound attenuation resulting from scattering and absorption of the wave by the tissues. Absorption of ultrasound in homogenous media occurs mostly as a result of relaxation losses and partially from internal friction and thermal conductivity, and increase in general with frequency [31].

The effectiveness of ultrasound heating can be improved by using sonosensitizers in the form of solid nanoparticles. The interaction between the ultrasonic wave and nanoparticles suspended in the continuous phase of medium (tissues) leads to the higher attenuation of the ultrasound wave compared to the attenuation in the continuous phase alone [32,33]. In our previous works we showed that the presence of nanoparticles leads to the additional attenuation of ultrasound and in consequence to the higher temperature increase $[9,20]$. Moreover, the valuable advantage of ultrasonic waves is that they can be focused in a small area and the depth of their penetration can be controlled. Therefore focused ultrasound heating can induce precise hyperthermia [34-36].

The experimental results of temperature changes vs time for agar phantom embedded with $0.43 \%$-concentration of MNPs at different acoustic powers are presented in Fig. 7 . The results demonstrate that in the first seconds temperature increases linearly with time and higher acoustic power causes faster growth of temperature. However, after about $5 \mathrm{~s}$ a steady state is achieved since the energy gain from the external source (ultrasound) is balanced by energy loss, mainly from heat conduction: the heat from the very small and hot focal zone of ultrasound (inset in Fig. 7) is transferred outside. That is why temperature stabilizes and saturates. 


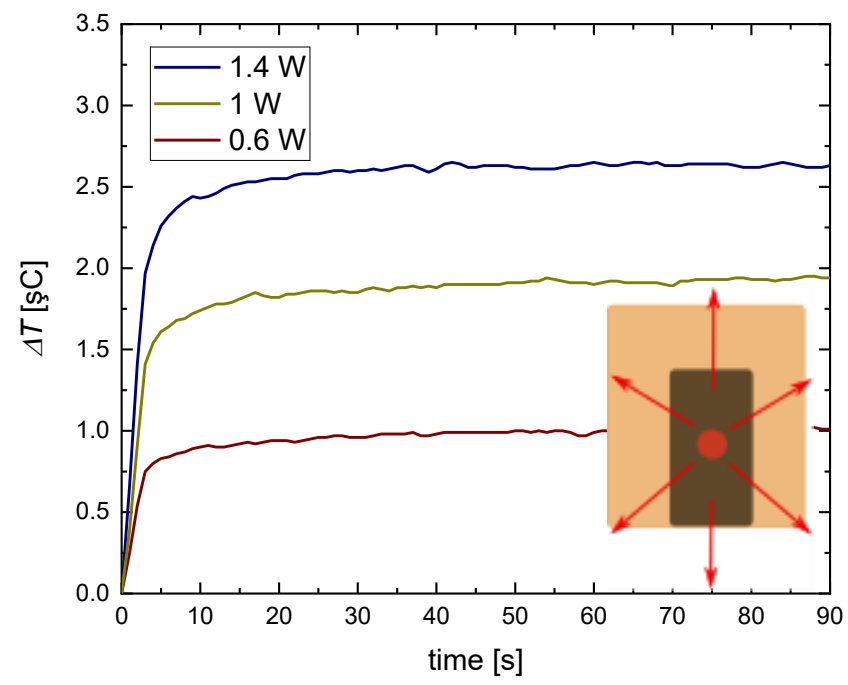

Fig. 7. Ultrasonic heating as a function of time and acoustic power. Inset: the ultrasound focal zone becomes the source of heat in phantom.

The nanoparticles used in magnetic heating have superparamagnetic properties. When magnetic nanoparticles placed in tumor tissue are subjected to an alternating magnetic field, the magnetic energy is transformed into heat. In case of superparamagnetic nanoparticles the only mechanism responsible for the heat generation is relaxation. This mechanism has two variants: Néel relaxation and Brown relaxation. The nanoparticle magnetic moment tends to align with the direction of the external magnetic field through the movement of the magnetic moment within a nanoparticle (Néel relaxation) or the rotation of the whole nanoparticle (Brown relaxation) [37, 38]. The temperature variations with time for the phantom with $0.43 \%$-concentration of MNPs for different values of the magnetic field strength during magnetic heating are presented in Fig. 8.

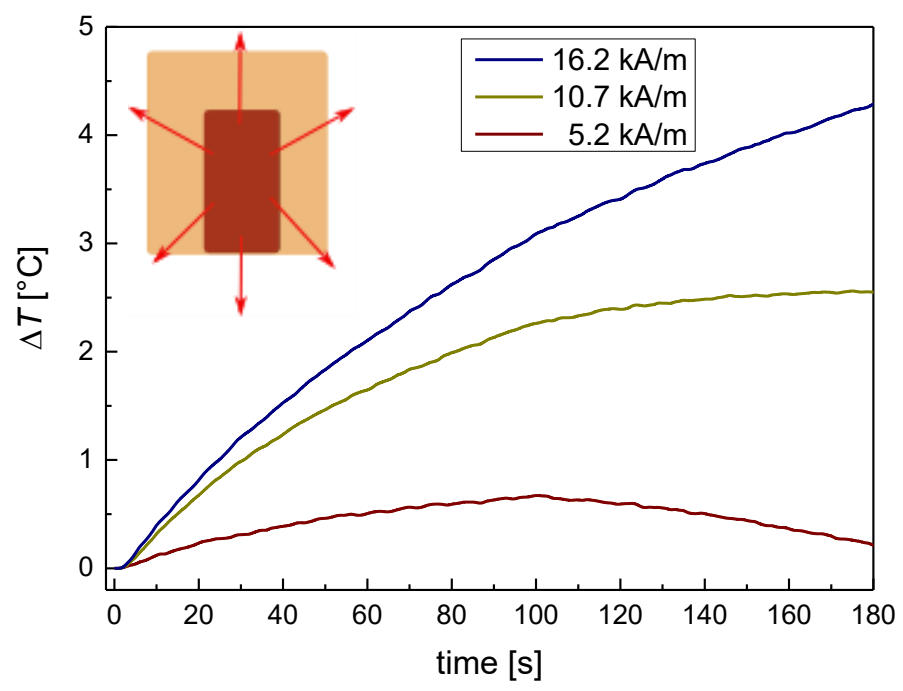

Fig. 8. Magnetic heating as a function of time and magnetic field strength. Inset: tumor phantom embedded with nanoparticles placed in the AMF becomes the source of heat. 
The results obtained demonstrate that with increasing strength of the magnetic field the higher temperature rise can be achieved. However, the initial temperature growth rate is much smaller than in case of ultrasonic heating, e.g., $0.0135 \mathrm{~K} / \mathrm{s}$ for magnetic heating with magnetic field strength of $16.2 \mathrm{kA} / \mathrm{m}$ and $0.25 \mathrm{~K} / \mathrm{s}$ for ultrasonic heating with the smallest acoustic power of $0.6 \mathrm{~W}$. Moreover, the curve for low magnetic field strength $(5.2 \mathrm{kA} / \mathrm{m})$ tends to bend downward. This is because more heat is transferred to surroundings (as healthy tissues or as in our case to pure agar phantom surrounding tumor model) than generated by magnetic nanoparticles. In clinical conditions the temperature achieved during the heating therapy can be substantially lowered by blood flow and blood perfusion. Blood perfusion through the vascular network influences the local temperature distribution thus changing the temperatures of the blood and tissue, which leads to convective heat transport [39]. Therefore, for low magnetic field strength it is difficult to maintain high-temperature increase and keep it stable over time. The reason for the weak temperature growth in tumor phantom during magnetic heating is the blockage of a Brown mechanism by the firm structure of gel (tissues).

One possibility is to use light (photo-induced hyperthermia) [40] or ultrasound which can be applied independently as a second source of heat $[9,11]$. The big advantage of ultrasound comparing to light wave is that FUS therapy reaches areas localized deep within the body [41]. The penetration depth of ultrasound in tissue can be controlled by appropriate choice of frequency. For $1 \mathrm{MHz}$ ultrasound penetration depth is about $20 \mathrm{~cm}$ and it increases for lower ultrasound frequencies [42, 43]. Whereas light can only be used if the tumor is not located deep in the body [40].

\subsection{Sono-magnetic heating}

The effectiveness of magnetic and ultrasonic heating used separately as a single modes of heating can be improved, as was shown in the previous section, by the use of the appropriate solid nanoparticles. They can noticeably enhance the thermal effect of the FUS heating due to the increase of ultrasound attenuation. Moreover, if they possess magnetic properties they will become the source of heat in alternating magnetic field. Therefore, magnetic nanoparticles constitute the perfect all-in-one material for bimodal heating. The combination of magnetic and ultrasound heating is a very promising approach. The bimodal therapy gives not only a cumulative heating in the site of interest but also creates conditions for synergistic interaction between the two mechanisms, namely the ultrasound sonication can improve the thermal effect of magnetic hyperthermia through the unblocking Brown's relaxation mechanism [11].

In our previous work [15] we showed that the effectiveness of magnetic heating depends on MNPs ability to rotate. However, MNPs movement in the gel structure of phantom (tissue) is limited. Therefore we assume that during this double heating ultrasound sonication increases the temperature and expands the pores of the phantom, which leaves more space for Brownian motions of nanoparticles, normally blocked in gel structure of phantom (tissues). This results in the observable additional temperature increase during magneto-ultrasonic heating.

During magneto-ultrasonic experiments the tumor phantom, immersed in distilled water, was simultaneously sonicated by ultrasound and exposed to the alternating magnetic field (Fig. 2). Fig. 9 presents the results of ultrasonic heating, magnetic heating and sono-magnetic heating for the tissuemimicking phantom with tumor model. 


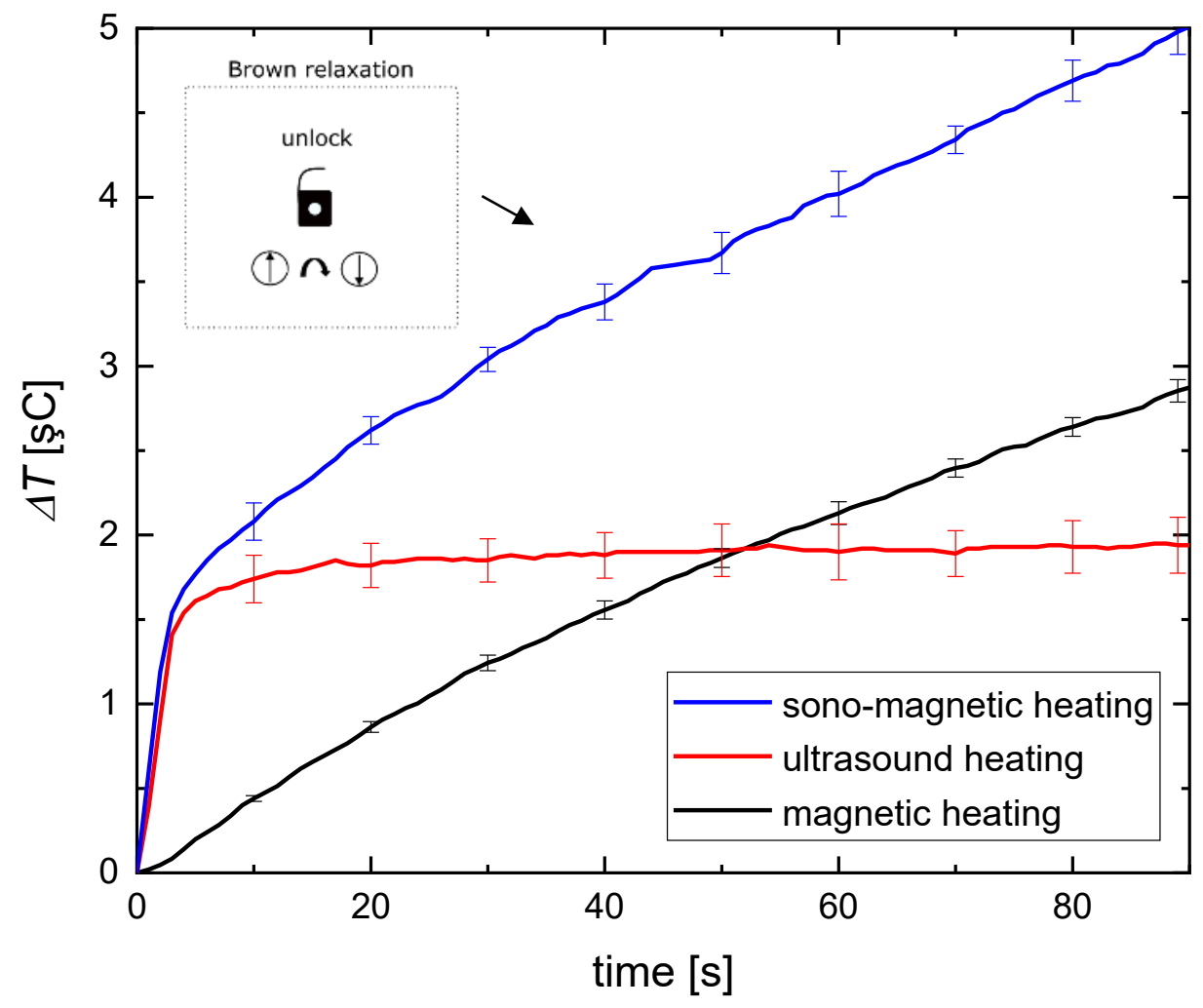

Fig. 9. Magnetic, ultrasonic and sono-magnetic heating as a function of time. Parameters: magnetic field strength $16.2 \mathrm{kA} / \mathrm{m}$, ultrasound frequency $1 \mathrm{MHz}$ with $1 \mathrm{~W}$ of acoustic power. Inset: Unlocking of the Brown relaxation mechanism is responsible for the observed increase in temperature during sono-magnetic heating.

The results of sono-magnetic heating demonstrate that the simultaneous action of ultrasound and magnetic field is more effective. It allows for achieving a better and faster heating comparing to the heating induced by single hyperthermia modalities. The ultrasound and magnetic heating are complementary to each other. The temperature rise caused by ultrasound is fast changing and by magnetic field slow changing. So the parameters of ultrasound can serve as coarse-tuning settings of heating while the parameters of magnetic field as fine-tuning enabling more precise hyperthermia.

The magnetic core also provides the hybrids with MRI detection (theranostic particles), allowing an in-depth imaging [40].

\section{Conclusion}

If we are to consider the application of magnetic hyperthermia on human tissues, experiments should be performed in conditions similar to the human body. In fluid suspensions, magnetic particles move freely and easily generate heat, however, in tissue-mimicking phantoms with mechanical properties that differ from fluids nanoparticle movement is limited and consequently less heat is generated. Moreover, if we are using tissue-mimicking phantoms or perform experiments on real tissues containg tumor we need to take into account the fact that healthy tissue surrounding cancerous one receives This is a peer-reviewed, accepted author manuscript of the following article: Kaczmarek, K., Hornowski, T., Antal, I., Rajnak, M., Timko, M., \& Józefczak, A. (2020). Sono-magnetic heating in tumor phantom. Journal of Magnetism and Magnetic Materials , 500, [166396]. https://doi.org/10.1016/j.jmmm.2020.166396 
heat and it is difficult to maintain high-temperature increase and keep it stable over time. However, the effectiveness of magnetic heating can be improved by merging it with other heating methods. In our work, we successfully improved magnetic heating by combining it with focused-ultrasounds and performed this bimodal treatment on tissue-mimicking phantoms with tumor model, placed inside, of different mechanical properties than healthy tissue. However, it is of importance to carry out, in the future, sono-magnetic experiments on real tissues. This bimodal heating appears to be more effective than magnetic or ultrasonic heating alone. In this bimodal heating, we can achieve the desired temperature increase in a shorter time than for magnetic or ultrasonic heating applied alone. Moreover, the double thermal stimulation of phantoms embedded with nanoparticles enables more precise control over the heating process and is a promising approach to treat cancer at lower nanoparticle concentrations and with shorter exposure times. The results show the potential of sonomagnetic heating for cancer treatments which comparing to other methods like photo-thermal therapy is able to reach tissues and areas localized deep within the body. Hopefully, in the future, it will help to create a new heating method suitable for thermal anticancer therapy.

Acknowledgments: This work was partly supported by the grants 2015/17/B/ST7/03566 and 2017/27/N/ST7/00201 of the Polish National Science Centre and the Slovak Research and Development Agency under the Contract No. APVV-15-0438. The author wishes to thank Mr. Wojciech Słoniewski for help in part of experimental research and dr. hab. Bernadeta Dobosz for help in USG imaging.

\section{References}

[1] G.-Y. Wan, Y. Liu, B.-W. Chen, Y.-Y. Liu, Y.-S. Wang, N. Zhang, Recent Advances of Sonodynamic Therapy in Cancer Treatment, Cancer Biol. Med. 13 (2016) 325-338.

[2] B. Fadeel, Hide and Seek: Nanomaterial Interactions With the Immune System, Frontiers in Immunology 10(133) (2019).

[3] K. Kaczmarek, T. Hornowski, R. Bielas, D. Żak, M. Timko, A. Józefczak, Dependence of Ultrasonic and Magnetic Hyperthermia on the Concentration of Magnetic Nanoparticles, Acta Phys. Pol. A 133(4) (2018) 716-718.

[4] A. de Leon, R. Perera, P. Nittayacharn, M. Cooley, O. Jung, A.A. Exner, Ultrasound Contrast Agents and Delivery Systems in Cancer Detection and Therapy, Adv. Cancer Res., Academic Press2018.

[5] A. Espinosa, R. Di Corato, J. Kolosnjaj-Tabi, P. Flaud, T. Pellegrino, C. Wilhelm, Duality of Iron Oxide Nanoparticles in Cancer Therapy: Amplification of Heating Efficiency by Magnetic Hyperthermia and Photothermal Bimodal Treatment, ACS Nano 10(2) (2016) 2436-2446.

[6] H. Chen, Y. Di, D. Chen, K. Madrid, M. Zhang, C. Tian, L. Tang, Y. Gu, Combined chemo- and photothermal therapy delivered by multifunctional theranostic gold nanorod-loaded microcapsules, Nanoscale 7(19) (2015) 8884-8897.

[7] R. Mendes, P. Pedrosa, J.C. Lima, A.R. Fernandes, P.V. Baptista, Photothermal enhancement of chemotherapy in breast cancer by visible irradiation of Gold Nanoparticles, Scientific Reports 7(1) (2017) 10872.

[8] B. Hildebrandt, P. Wust, O. Ahlers, A. Dieing, G. Sreenivasa, T. Kerner, R. Felix, H. Riess, The cellular and molecular basis of hyperthermia, Critical Reviews in Oncology/Hematology 43(1) (2002) 33-56.

[9] K. Kaczmarek, T. Hornowski, M. Kubovčíková, M. Timko, M. Koralewski, A. Józefczak, Heating Induced by Therapeutic Ultrasound in the Presence of Magnetic Nanoparticles, ACS Applied Materials \& Interfaces 10(14) (2018) 11554-11564.

[10] S. Dutz, R. Hergt, Magnetic particle hyperthermia-a promising tumour therapy?, Nanotechnology 25(45) (2014) 452001.

This is a peer-reviewed, accepted author manuscript of the following article: Kaczmarek, K., Hornowski, T., Antal, I., Rajnak, M., Timko, M., \& Józefczak, A. (2020). Sono-magnetic heating in tumor phantom. Journal of Magnetism and Magnetic Materials , 500, [166396]. https://doi.org/10.1016/j.jmmm.2020.166396 
[11] K. Kaczmarek, T. Hornowski, I. Antal, M. Timko, A. Józefczak, Magneto-ultrasonic heating with nanoparticles, J. Magn. Magn. Matter. 474 (2019) 400-405.

[12] D.L. Miller, N.B. Smith, M.R. Bailey, G.J. Czarnota, K. Hynynen, I.R.S. Makin, B.C.o.t.A.I.o.U.i. Medicine, Overview of Therapeutic Ultrasound Applications and Safety Considerations, Journal of Ultrasound in Medicine 31(4) (2012) 623-634.

[13] S. Ahmad Reza Dibaji, M.F. Al-Rjoub, M.R. Myers, R.K. Banerjee, Enhanced Heat Transfer and Thermal Dose Using Magnetic Nanoparticles During HIFU Thermal Ablation-An In-Vitro Study, Journal of Nanotechnology in Engineering and Medicine 4(4) (2014) 040902-040902.

[14] R.S. Molday, D. Mackenzie, Immunospecific ferromagnetic iron-dextran reagents for the labeling and magnetic separation of cells, Journal of Immunological Methods 52(3) (1982) 353-367.

[15] K. Kaczmarek, R. Mrówczyński, T. Hornowski, R. Bielas, A. Józefczak, The Effect of TissueMimicking Phantom Compressibility on Magnetic Hyperthermia, Nanomaterials 9(5) (2019) 803. [16] M. Zhang, Z. Che, J. Chen, H. Zhao, L. Yang, Z. Zhong, J. Lu, Experimental Determination of Thermal Conductivity of Water-Agar Gel at Different Concentrations and Temperatures, Journal of Chemical \& Engineering Data 56(4) (2011) 859-864.

[17] M. Salloum, R.H. Ma, D. Weeks, L. Zhu, Controlling nanoparticle delivery in magnetic nanoparticle hyperthermia for cancer treatment: Experimental study in agarose gel, International Journal of Hyperthermia 24(4) (2008) 337-345.

[18] M.O. Culjat, D. Goldenberg, P. Tewari, R.S. Singh, A Review of Tissue Substitutes for Ultrasound Imaging, Ultrasound in Medicine and Biology 36(6) (2010) 861-873.

[19] A. Dabbagh, B.J.J. Abdullah, C. Ramasindarum, N.H. Abu Kasim, Tissue-Mimicking Gel Phantoms for Thermal Therapy Studies, Ultrasonic Imaging 36(4) (2014) 291-316.

[20] A. Józefczak, K. Kaczmarek, M. Kubovčíková, Z. Rozynek, T. Hornowski, The effect of magnetic nanoparticles on the acoustic properties of tissue-mimicking agar-gel phantoms, J. Magn. Magn. Matter. 431 (2017) 172-175.

[21] J. Huang, R.G. Holt, R.O. Cleveland, R.A. Roy, Experimental validation of a tractable numerical model for focused ultrasound heating in flow-through tissue phantoms, The Journal of the Acoustical Society of America 116(4) (2004) 2451-2458.

[22] B. Silver, T.S. Metzger, T.A. Matalon, A simple phantom for learning needle placement for sonographically guided biopsy, American Journal of Roentgenology 154(4) (1990) 847-848.

[23] J.W. Li, M.K. Karmakar, X. Li, W.H. Kwok, W.D.N. Kee, Gelatin-Agar Lumbosacral Spine Phantom, Journal of Ultrasound in Medicine 30(2) (2011) 263-272.

[24] H. Saraf, K.T. Ramesh, A.M. Lennon, A.C. Merkle, J.C. Roberts, Mechanical properties of soft human tissues under dynamic loading, Journal of Biomechanics 40(9) (2007) 1960-1967.

[25] M.J. Paszek, N. Zahir, K.R. Johnson, J.N. Lakins, G.I. Rozenberg, A. Gefen, C.A. Reinhart-King, S.S. Margulies, M. Dembo, D. Boettiger, D.A. Hammer, V.M. Weaver, Tensional homeostasis and the malignant phenotype, Cancer Cell 8(3) (2005) 241-254.

[26] S.-L. Xue, S.-Z. Lin, B. Li, X.-Q. Feng, A nonlinear poroelastic theory of solid tumors with glycosaminoglycan swelling, Journal of Theoretical Biology 433 (2017) 49-56.

[27] M. Plodinec, M. Loparic, C.A. Monnier, E.C. Obermann, R. Zanetti-Dallenbach, P. Oertle, J.T. Hyotyla, U. Aebi, M. Bentires-Alj, R.Y.H. Lim, C.-A. Schoenenberger, The nanomechanical signature of breast cancer, Nature Nanotechnology 7 (2012) 757-765.

[28] F. Davidson, Ultrasonic Power Balances, in: R.C. Preston (Ed.), Output Measurements for Medical Ultrasound, Springer London, London, 1991, pp. 75-90.

[29] Z. Rozynek, A. Józefczak, K.D. Knudsen, A. Skumiel, T. Hornowski, O. Fossum, M. Timko, P. Kopčanský, M. Koneracká, Structuring from nanoparticles in oil-based ferrofluids, Eur. Phys. J. E 34 (2011) 28.

[30] K. Kaczmarek, T. Hornowski, B. Dobosz, A. Józefczak, Influence of Magnetic Nanoparticles on the Focused Ultrasound Hyperthermia, Materials 11(9) (2018) 1607.

[31] J.C. Bamber, Attenuation and Absorption, in: C.R. Hill, J.C. Bamber, R.t. Haar (Eds.), Physical Principles of Medical Ultrasonics2005, pp. 93-166.

This is a peer-reviewed, accepted author manuscript of the following article: Kaczmarek, K., Hornowski, T., Antal, I., Rajnak, M., Timko, M., \& Józefczak, A. (2020). Sono-magnetic heating in tumor phantom. Journal of Magnetism and Magnetic Materials , 500, [166396]. https://doi.org/10.1016/i.jmmm.2020.166396 
[32] T.J. Dubinsky, C. Cuevas, M.K. Dighe, O. Kolokythas, J.H. Hwang, High-Intensity Focused Ultrasound: Current Potential and Oncologic Applications, American Journal of Roentgenology 190(1) (2008) 191-199.

[33] H. ter, gt, Gail, C. Coussios, High intensity focused ultrasound: Physical principles and devices, International Journal of Hyperthermia 23(2) (2007) 89-104.

[34] N.P.K. Ellens, K. Hynynen, 22 - High-intensity focused ultrasound for medical therapy, in: J.A. Gallego-Juárez, K.F. Graff (Eds.), Power Ultrasonics, Woodhead Publishing, Oxford, 2015, pp. 661693.

[35] D.B. Miller, J.P. O'Callaghan, New horizons for focused ultrasound (FUS) \&\#x2013; therapeutic applications in neurodegenerative diseases, Metabolism - Clinical and Experimental 69 (2017) S3-S7. [36] R.J. Piper, M.A. Hughes, C.M. Moran, J. Kandasamy, Focused ultrasound as a non-invasive intervention for neurological disease: a review, British Journal of Neurosurgery 30(3) (2016) 286-293. [37] D. Silvio, H. Rudolf, Magnetic particle hyperthermia-a promising tumour therapy?, Nanotechnology 25(45) (2014) 452001.

[38] A.E. Deatsch, B.A. Evans, Heating efficiency in magnetic nanoparticle hyperthermia, Journal of Magnetism and Magnetic Materials 354 (2014) 163-172.

[39] R.K. Jain, Analysis of Heat Transfer and Temperature Distributions in Tissues during Local and Whole-Body Hyperthermia, in: A. Shitzer, R.C. Eberhart (Eds.), Heat Transfer in Medicine and Biology: Analysis and Applications. Volume 2, Springer US, Boston, MA, 1985, pp. 3-54.

[40] A. Curcio, A.K.A. Silva, S. Cabana, A. Espinosa, B. Baptiste, N. Menguy, C. Wilhelm, A. AbouHassan, Iron Oxide Nanoflowers @ CuS Hybrids for Cancer Tri-Therapy: Interplay of Photothermal Therapy, Magnetic Hyperthermia and Photodynamic Therapy, Theranostics 9(5) (2019) 1288-1302. [41] T.J. Dubinsky, C. Cuevas, M.K. Dighe, O. Kolokythas, J.H. Hwang, High-intensity focused ultrasound: current potential and oncologic applications, American Journal of Roentgenology 190 (2008) 191-199.

[42] N.P.K. Ellens, K. Hynynen, 22 - High-intensity focused ultrasound for medical therapy A2 Gallego-Juárez, Juan A, in: K.F. Graff (Ed.), Power Ultrasonics, Woodhead Publishing, Oxford, 2015, pp. 661-693.

[43] R.B. Roemer, Ultrasonic Heating Techniques, in: S.B. Field, C. Franconi (Eds.), Physics and Technology of Hyperthermia, Springer Netherlands, Dordrecht, 1987, pp. 390-402. 